\title{
Subcutaneous IgG Replacement Therapy by Push in 32 Patients with Primary Immunodeficiency Diseases in Argentine
}

\author{
Bezrodnik Liliana ${ }^{1 *}$, Gómez Raccio Andrea ${ }^{1}$, Regairaz Lorena ${ }^{2}$, Díaz Ballve Damacia ${ }^{1}$, Seminario Gisela , Moreira lleana ${ }^{1}$ and Di Giovanni \\ Daniela ${ }^{1}$
}

${ }^{1}$ Inmunología, Hospital de Niños “Dr. Ricardo Gutiérrez”, Bs. As. Argentina

${ }^{2}$ Inmunología, Hospital de Niños "Sor María Ludovica”, La Plata. Argentina

\begin{abstract}
Introduction: Regular replacement with immunoglobulin infusions is the mainstay of treatment in the majority of primary immunodeficiencies. Several studies showed that Subcutaneous Immunoglobulin (SCIG) has similar efficacy to Intravenous Immunoglobulin (IVIG) in preventing infections in PID patients. Here we report effectiveness, safety and tolerance of SCIG replacement therapy by push in 32 pediatric and adult patients with humoral PID in Argentina.

Results: We describe 32 patients that received SCIG treatment between July 2011 and May 2012. 17 male and 15 female from 2 Immunology Centers; aged from 8 months to 40 years (median: 11 years). 30 patients previously received IVIG treatment. Among them, fifteen received 9 months of SCIG treatment administered by pump. The other 2 patients started the immunoglobulin replacement treatment directly with SCIG by push. The mean dose of SCIG was $133 \mathrm{mg} / \mathrm{kg} /$ week (range 100-192). The annual rate of any infection was 1, 2 infection/year/patient for subcutaneous treatment. The frequency of adverse effects was $0.02 \%$ with the SCIG. At the end of the study, all patients chose SCIG home-therapy regimen and referred much more comfort with SCIG by push.
\end{abstract}

Conclusion: Self-administered subcutaneous immunoglobulin therapy by push is an effective and safe alternative therapy for patients with PID.

Keywords: Primary immunodeficiencies; Subcutaneous gammaglobulin; Push self-administration

\section{Introduction}

Primary Immunodeficiency Diseases (PID) comprise more than 200 different congenital disorders that affect the development, function, or both of the immune system, leading to increased frequency of infections, mainly bacterial infections of the respiratory tract [1-3]. Regular replacement with immunoglobulin infusions is the mainstay of treatment in the majority of primary immunodeficiencies, both in antibody deficiencies and in severe $\mathrm{T}$ cells deficiencies $[4,5]$.

Subcutaneous Infusions of Immunoglobulin (SCIG) were introduced in 1980 in US and since then, they have been increasingly used in this country and in Europe [6]. Several studies have shown that SCIG has similar efficacy to Intravenous Immunoglobulin (IVIG) in preventing infections in PID patients [7-14]. The low risk of systemic adverse reactions makes the subcutaneous route for IgG administration a good choice for home-therapy and an excellent alternative to patients with poor venous access or severe adverse effects with IVIG $[7,15,16]$.

Beriglobina P MR (CSL Behring), a preparation of human immunoglobulin to be administered subcutaneously, was approved by the Argentinian Health Department, Secretaría de Políticas, Regulación y Relaciones Sanitarias A.N.M.A.T. in 2010 (www.anmat.gov.ar). Since then, we have started using SCIG as replacement therapy and we have made a multicentric study with 15 pediatric patients with PID during nine months to compare SCIG effectiveness, safety and tolerance, administered by pump vs. IVIG [17]. According to the improvement of the serum IgG levels reached with SCIG infusions, the reduction of infectious episodes and the decrease of moderate and severe adverse events with this kind of treatment, we continued prescribing replacement therapy with SCIG in more children and adult patients with humoral PID but we are still dealing with the difficulties related to the pump costs. Shapiro et al. [18] showed the feasibility to use the SCIG therapy by push, and for that reason we started administrating SCIG by push since July 2011.
Here we report effectiveness, safety and tolerance of SCIG replacement therapy by push in 32 pediatric and adult patients with humoral PID in Argentine.

\section{Material and Methods}

\section{Study design}

This is an observational, descriptive and ambispective study. The objective is to describe the effectiveness, safety and tolerance of SCIG therapy by push in patients that received this treatment in our center between July 2011 and May 2012.

Baseline data, including steady-state serum IgG levels, bacterial infections, and adverse reactions, were obtained from the medical records.

This protocol was approved by the ethics committee for each hospital and written informed consent was obtained.

\section{Patients}

To be included in the study, patients must have a Humoral PID diagnosis that required immunoglobulin replacement treatment. Patients with chronic infections like Hepatitis-B Virus (HBV), Hepatitis-C Virus (HCV) and Human Immunodeficiency Virus-1 (HIV-1) were excluded.

*Corresponding author: Liliana Bezrodnik, CABA, Argentina, Tel/Fax: 54011 47859844; E-mail: Ibezrodnik@yahoo.com.ar

Received December 21, 2013; Accepted January 17, 2014; Published January 24, 2014

Citation: Liliana B, Andrea GR, Lorena R, Damacia DB, Gisela S, et al. (2014) Subcutaneous IgG Replacement Therapy by Push in 32 Patients with Primary Immunodeficiency Diseases in Argentine. Clin Exp Pharmacol 4: 148. doi:10.4172/2161-1459.1000148

Copyright: ( 2014 Liliana B, et al. This is an open-access article distributed under the terms of the Creative Commons Attribution License, which permits unrestricted use, distribution, and reproduction in any medium, provided the original author and source are credited. 
The diagnosis of Humoral PID was one of the following: X-linked Agammaglobulinemia (XLA) (Diagnostic criteria: ESID/PAGID 1999) [19], Common Variable Immunodeficiency (CVID) (Diagnostic criteria: ESID/PAGID 1999) [19], Hyper IgM Syndrome (SHIM) (Diagnostic criteria: $\operatorname{IgG}<2 \mathrm{SD}$, absent $\operatorname{IgA}, \operatorname{IgM}>2 \mathrm{SD}$ and normal number of $\mathrm{B}$ lymphocyte) in one woman, Severe Hipogammaglobulinemia (SH) (Diagnostic criteria: Serum IgG $<300 \mathrm{mg} \%$ and B Lymphocyte $>2 \%$ ) or Specific Antibody Deficiency (SAD) (Diagnostic criteria: Patients with impaired response to pneumococcal antigens with the 23-valent pneumococcal vaccine and normal IgG values per age) [20].

Bronchiectasis was defined as an abnormal and irreversible dilatation of the bronchi, frequently associated with inflammation.

The protocol was approved by the independent ethics committee for each hospital and written informed consent was obtained for each subject or their parents if they were under 18 years old. The study was performed in accordance with the Declaration of Helsinki and Good Clinical Practice Guidelines as well as any applicable local regulations.

\section{Treatment: Doses and SCIG infusion technique}

We used Beriglobina P (CSL Behring), ready-to-use pasteurized preparation of human polyvalent immunoglobulin to be administered subcutaneously, at a concentration of $16 \%(160 \mathrm{mg} / \mathrm{ml})$.

If the patient was receiving IVIG treatment previously, weekly dosage of SCIG was calculated by dividing the previous IVIG monthly dose by 4 , so the patients received in the SCIG therapy the same dose as in the IVIG therapy. The administration route was switched 7 days after the last intravenous infusion. The patients that had started the immunoglobulin treatment with SCIG received weekly infusions of SCIG at a dose of $100-150 \mathrm{mg} / \mathrm{kg} /$ week.

A period of 4-6 training weeks in hospital before home selfadministration was provided. The family member in charge or the own patient learned about the use of premedication, the technique of application and instructions to complete the register with details of each home infusion, infections and adverse reactions.

The product was administered at 1 or multiple injection sites, by push with rates limited to $1 \mathrm{ml} / \mathrm{min}$ and in each site a maximum of 20 $\mathrm{ml}$ in children and $35 \mathrm{ml}$ in adults.

\section{Physical examination and blood assessments}

Physical investigations were performed each time the patient visited the clinic. Vital signs (blood pressure, heart rate, respiratory rate, and body temperature) were recorded before and during infusion at first 4-6 weeks (training sessions).

Serum immunoglobulin (IgG, IgA, and IgM) (Method: Kinetic Nephelometry-Immunochemistry System IMMAGE, BeckmanCoulter), hematological status and clinical chemistry profile were

\begin{tabular}{|c|c|c|c|}
\hline \multirow{2}{*}{ Sex } & & Frequency & $\%$ \\
\hline \multirow{4}{*}{ Diagnosis } & Male & 17 & $53.1 \%$ \\
\cline { 2 - 4 } & Female & 15 & $46.9 \%$ \\
\cline { 2 - 4 } & SH & 2 & $6.2 \%$ \\
\cline { 2 - 4 } & CVID & 5 & $15.6 \%$ \\
\cline { 2 - 4 } & SAD & 12 & $37.5 \%$ \\
\hline \multirow{2}{*}{ Bronchiectasis } & SHIM & 1 & $37.5 \%$ \\
\cline { 2 - 4 } & Yes & 13 & $3.1 \%$ \\
\hline \multirow{2}{*}{$*$} & No & 19 & $40.6 \%$ \\
\hline
\end{tabular}

Table 1: Patient demographic and baseline characteristics. determined before the infusions at weeks $2,4,8,16,24$ and 36 in all patients. Viral tests were done on patient blood samples before starting the SCIG treatment and at the end of the study and included Hepatitis-B surface antigen, polymerase chain reaction HCV and HIV-1.

\section{Effectiveness}

Effectiveness was measured by IgG levels and the number and characteristics of bacterial infections. Serious bacterial infection had been defined as meningitis, sepsis, osteomyelitis or visceral abscesses according to the definition of the US Food and Drug Administration [21].

The immunologist in charge of the patient documented the characteristics of infections.

At the end of study, patients were asked about what kind of IgG regimen infusion they preferred to continue with IVIG, SCIG by pump or SCIG by push.

\section{Tolerance}

Tolerance was evaluated by the number and type of Adverse Events (AEs). They were classified in mild (local erythema, swelling, pruritus or pain for more than 24 hrs after the infusion), moderate (headache, fever, vomiting, urticaria) and severe (cyanosis, meningism or other sign of life threatening); local AEs (infusion-site reaction) and systemic AEs (treatment related and no infusion-site reaction).

\section{Statistical analysis}

Data was analyzed using descriptive statistics (mean, Standard Deviation [SD], median, and minimum and maximum). Frequencies and percentages were calculated for the qualitative variables; these were compared using the chi-squared independence test. When quantitative variables (IgG dosages) were compared in different weeks, a repeated measure analysis of variance (ANOVA) was applied. In all cases $<5 \%$ was used to reject the null hypothesis (no difference).

\section{Results}

\section{Patients}

In the period of study, from July 2011 to May 2012, 32 patients from two Immunology Centers started SCIG treatment: 2 X-linked Agammaglobulinemia, 12 Common Variable Immunodeficiency, 1 Hyper IgM Syndrome, 5 Severe Hypogammaglobulinemia and 12 Specific Antibody Deficiency.

They were 17 male and 15 female; aged from 8 months to 40 years (median 11 years). 13 patients had documented bronchiectasis before the beginning of the SCIG treatment (Table 1).

There were 30 patients that had previously received IVIG treatment, for at least 48 weeks and all of them presented stable IgG serum levels $(>500 \mathrm{mg} / \mathrm{dl})$. In this patients $\mathrm{IgG}$ serum levels were dosed every 4 months. Among them, 15 patients had received first IVIG and after that 9 months of SCIG treatment administered by pump. The other 2 patients started the immunoglobulin replace treatment directly with SCIG.

For all patients, hematological and clinical chemistry profile results were normal and infectious studies were negatives.

\section{Doses and SCIG infusion technique}

The mean dose of SCIG was $133 \mathrm{mg} / \mathrm{kg} /$ week (range 100-192). Prophylactic antihistamines were prescribed to all patients at the 


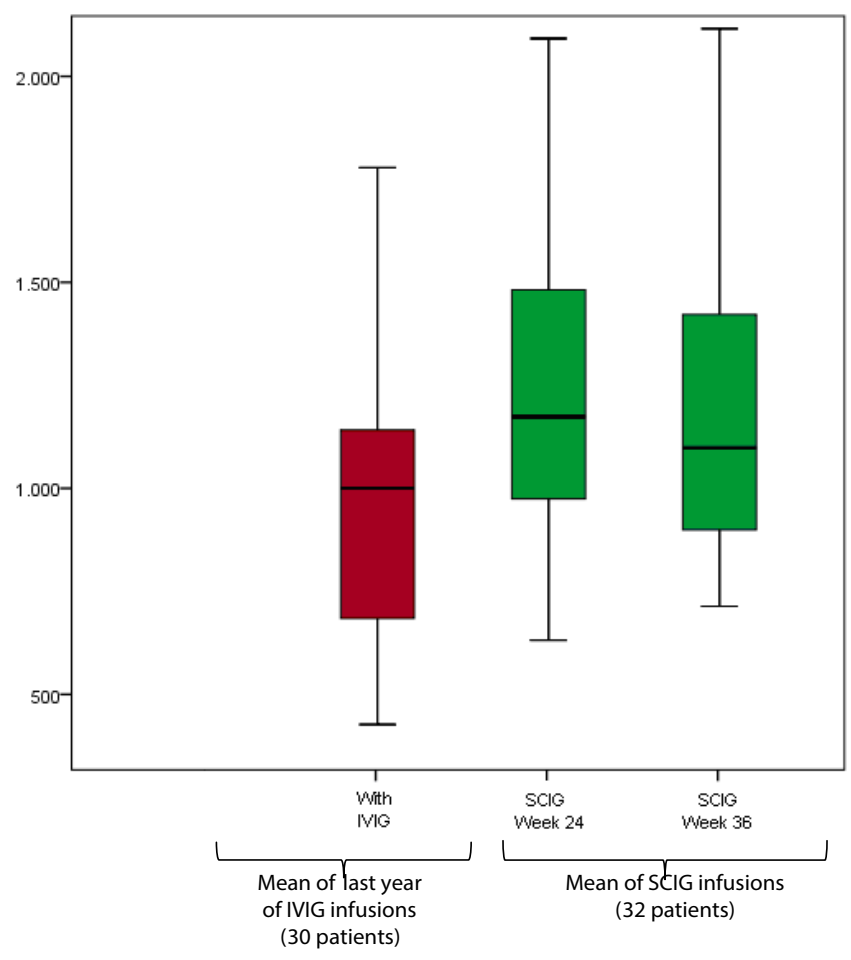

An increase in IgG plasma concentration was observed following the switch from IVIG to SCIG administration. Boxes represent 25 and 75 percentile values. Error bars represent the 5 and 95 percentile values.

Figure 1: Median IgG plasma concentration before and during SCIG replacement therapy.

\begin{tabular}{|c|c|c|}
\hline & Mean & Standard deviation \\
\hline $\begin{array}{c}\text { 12 months prior to SCIG treatment } \\
\text { (30 patients with IVIG) }\end{array}$ & 1005.33 & 419.420 \\
\hline SCIG week 24 (32 patients) & 1263.60 & 534.638 \\
\hline SCIG week 36 (32 patients) & 1205.47 & 457.990 \\
\hline
\end{tabular}

Table 2: Serum IgG levels before and during SCIG replacement therapy.

beginning of the SCIG treatment but at the end of the study only $78 \%$ (25/32) chose to continue taking this treatment.

The abdomen was used as injection site in 29/32 patients and the thigh was used in $3 / 32$ with good tolerance. 2 infusion sites were used $18 / 32$ patients, 1 site was used in $13 / 32$, and 3 sites per session were used in $1 / 32$ patient.

\section{Effectiveness}

Measurement IgG levels in IVIG and SCIG therapy: In 30 patients that had previously received IVIG, an increase in IgG plasma concentration was observed following the switch from IVIG to SCIG administration (Figure 1). The mean serum IgG level during the last 48 weeks of the IVIG treatment was 1005 (419) $\mathrm{mg} / \mathrm{dl}$ compared to $1264(535) \mathrm{mg} / \mathrm{dl}$ at week 24, 1205 (458) $\mathrm{mg} / \mathrm{dl}$ at week 36 with SCIG treatment (Table 2). There was a statistically significant increase in the mean IgG level at weeks 24 and 36 compared to the mean IgG level during IVIG treatment $(\mathrm{p}<0.001)$.

Infections (number, type and severity): With SCIG treatment, 16 of the 32 patients (50\%) presented 32 ambulatory infection episodes during the period of study (48 weeks): otitis media, bronchitis, pneumonia. Three patients presented a pneumonia episode that required hospital admission.

Among the group of patients that previously received IVIG during the last 48 weeks with IVIG, 18 patients $(60 \%)$ had 69 infection episodes: otitis media, sinusitis, bronchitis, pneumonias; five patients presented an infection that required hospital admission (4 pneumonias, 1 bronchitis).

The annual rate of all infections (ambulatory plus hospitalized) was 2, 7 infections/year/patient for intravenous route compared to 1, 2 infections/year/patient for subcutaneous treatment.

13/32 patients had bronchiectasis with IVIG treatment, 39 lower respiratory infections episodes ( 27 bronchitis and 12 pneumonias) were suffered in five patients with bronchiectasis. With SCIG treatment, 10 lower respiratory infections episodes ( 9 bronchitis and 1 pneumonia) were diagnosed in two patients with bronchiectasis.

Tolerance (number and type of AEs): During the study period, 1152 SCIG infusion were administered. 6/32 patients reported 34 events of adverse reactions ( $34 / 1152$ infusions $=0.02$ adverse effect/infusion). Five patients presented 32 mild episodes related with the injection site and only one presented 2 moderate adverse reactions (headache).

Regarding the mild adverse reactions, five patients related 32 episodes that last for more than $24 \mathrm{hrs:} \mathrm{erythema} \mathrm{(10} \mathrm{episodes),} \mathrm{swelling}$ (8 episodes), itching (4 episodes) and local pain (10 episodes).

Among 30 patients with previous IVIG treatment, in the last 48 weeks, 270 IVIG infusions had been administered, 10/30 patients reported 36 events of adverse reactions ( $36 / 270$ infusions $=0.13$ adverse effect/infusion). One (3.3\%) patient referred 3 episodes of mild adverse effects related with the site of infusion (pain). Nine (30\%) patients reported 32 events of moderated adverse reaction with different IVIG products: 7 patients had headache ( 24 events), 3 patients had fever (4 events), 3 patients had vomits (4 events), and one patient presented 1 episode of severe adverse effect: dyspnea, cyanosis and meningism.

\begin{tabular}{|c|c|c|}
\hline Type of adverse event & $\begin{array}{l}\text { With IVlgG }{ }^{*} \text { treatment } \\
30 \text { patients }\end{array}$ & $\begin{array}{c}\text { With } \mathrm{SClgG}^{\dagger} \text { treatment } \\
32 \text { patients }\end{array}$ \\
\hline & $\begin{array}{l}\text { Episodes in numbers } \\
\text { (patients affected) }\end{array}$ & $\begin{array}{l}\text { Episodes in numbers } \\
\text { (patients affected) }\end{array}$ \\
\hline Mild & $3(1)$ & $32(5)$ \\
\hline Local Erithema & 0 & $10(4)$ \\
\hline Local Swelling & 0 & $8(2)$ \\
\hline Local Pain & $3(1)$ & $10(2)$ \\
\hline Local Pruritus/Eczema & 0 & $4(2)$ \\
\hline Moderate & $32(8)$ & $2(1)$ \\
\hline Headache & $24(7)$ & $2(1)$ \\
\hline Fever & $4(3)$ & 0 \\
\hline Vomiting & $4(3)$ & 0 \\
\hline Urticaria & 0 & 0 \\
\hline Severe & $1(1)$ & 0 \\
\hline $\begin{array}{l}\text { Dyspnea cyanosis } \\
\text { meningism }\end{array}$ & $1(1)$ & 0 \\
\hline Total episodes & $36(10)$ & $34(6)$ \\
\hline Total infusions & 270 & 1152 \\
\hline $\begin{array}{l}\text { Rate (episodes/ } \\
\text { infusion) }\end{array}$ & 0.13 & 0.02 \\
\hline
\end{tabular}

The reports of adverse effects of each patient have individual variations, considering the method of home self register.

*IVIgG: Intravenous Immunoglobuline G.

†SCIgG: Subcutaneous Immunoglobuline.

Table 3: Frequency and type of adverse events. 
At the end of the study we asked the patients which administration route they preferred to continue with the treatment and all of them chose the SCIG home-therapy regimen, based on weekly self-infusions.

Among 15 patients that had previously received SCIG treatment by pump, all of them preferred to continue the administration by push (Table 3).

\section{Discussion}

Appropriate treatment with IVIG or SCIG is the most important aspect of management for majority of individuals with PID. Different authors have reported a number of potential benefits of subcutaneous administration of immunoglobulin [11]. This study demonstrated the efficacy, safety and tolerability of self-administered subcutaneous immunoglobulin therapy by push in pediatric and adult patients with PID.

With the incorporation of therapeutic IVIG in PID in Argentina, we have seen a marked improvement in the quality of patient's life. Nowadays the possibility of using SCIG allow us to offer another treatment option to our patients. Our study evaluates the evolution of 32 pediatric and adult patients with antibody deficiencies which received SCIG between July 2011 and May 2012, 30 of which had previous received IVIG treatment.

The efficacy data collected during the study is similar to that reported from previous trials using other subcutaneously administered immunoglobulin preparations $[13,22,23]$.

According to what we described before in a multicentric study with 15 pediatric patients with PID comparing effectiveness, safety and tolerance with SCIG administered by pump vs. IVIG in Argentina [17], serum IgG levels during SCIG therapy reached therapeutic values. When we compared the IgG levels in IVIG and SCIG treatment, we observed that the median ratio of serum IgG levels during SCIG therapy was higher $(\mathrm{p}<0.001)$. The serum IgG levels kept constant during the evaluation period, there were no oscillations of serum IgG levels between consecutive SCIG infusions according to other publications [24-26].

Also, we observed a reduction of infectious events and requirement of hospitalization. Considering only 30 patients that had previously received IVIG, the number of ambulatory infections was reduced during the SCIG treatment in all patients. It is important to highlight that the lower respiratory tract infections decreased in patients with bronchiectasis. We hypothesize that the decrease in infectious processes could be a consequence of the higher serum IgG levels achieved with SCIG.

The benefits of SCIG include elimination of venous access, improvement and stabilization of serum IgG levels, less number and severity of infections and decreased systemic adverse reactions. The potential risk of life-threatening anaphylactic shock may be reduced or even eliminated.

Subcutaneous administration was generally well tolerated, with no systemic or clinically significant adverse reactions [13,22,23]. According to other publications almost every injection-site reaction was mild. Although some of the patients experienced treatment-related AEs, this was primarily due to infusion-site reactions including local erythema, swelling, itching and pain. In the different variables analyzed there were no significant differences in sex and age.

Quality-of-life studies have consistently shown that self infusion at home is crucial for patient satisfaction $[27,28]$. At the end of study and in order to continue with their treatment, all patients chosed the SCIG home-therapy regimen based on, weekly self-infusions among the ones that had received SCIG treatment by pump before. All of them referred to be more comfortable administering the SCIG by push. Most importantly, all participating patients managed, without further difficulties, the task of self infusion at home.

Concluding, the data from this study confirms that selfadministered, subcutaneous immunoglobulin therapy by push is an effective and safe alternative therapy for patients with PID.

\section{Conflict of Interest}

The professionals among the authors don't have conflict of interests with the company CSL Behring.

\section{References}

1. Stiehm ER, Ochs HD, Winkelstein JA (2004) Immunologic disorders: General considerations. WB Saunders, Philadelphia, USA 289-335.

2. International Union of Immunological Societies Expert Committee on Primary Immunodeficiencies, Notarangelo LD, Fischer A, Geha RS, Casanova JL, et al. (2009) Primary immunodeficiencies: 2009 update. J Allergy Clin Immunol 124: 1161-1178.

3. Notarangelo LD (2010) Primary immunodeficiencies. J Allergy Clin Immuno 125: S182-194.

4. Al-Herz W, Bousfiha A, Casanova JL (2011) Primary immunodeficiency diseases: an update on the classification from the International Union of Immunological Societies Expert Committee for Primary Immunodeficiency. Front Immunol 2: 1-26.

5. Chapel H (2011) Classification of primary immunodeficiency diseases by the International Union of Immunological Societies (IUIS) Expert Committee for Primary Immunodeficiency 2011. Clinical and Experimental Immunology, 168 58-59.

6. Berger M, Cupps TR, Fauci AS (1980) Immunoglobulin replacement therapy by slow subcutaneous infusion. Ann Intern Med 93: 55-56.

7. Berger M (2008) Subcutaneous administration of IgG. Immunol Allergy Clin North Am 28: 779-802, viii.

8. Gardulf A, Andersen V, Björkander J, Ericson D, Frøland SS, et al. (1995) Subcutaneous immunoglobulin replacement in patients with primary antibody deficiencies: safety and costs. Lancet 345: 365-369.

9. Abrahamsen TG, Sandersen H, Bustnes A (1996) Home therapy with subcutaneous immunoglobulin infusions in children with congenital immunodeficiencies. Pediatrics 98: 1127-1131.

10. Bhole MV, Burton J, Chapel HM (2008) Self-infusion programmes for immunoglobulin replacement at home: feasibility, safety and efficacy. Immunol Allergy Clin North Am 28: 821-832, ix.

11. Maroto Hernando $M$, Soler Palacin $P$, Nalda M, Oliveras Arenas M, Español Boren T, et al. (2009) Gammaglobulina subctutánea en inmunodeficiencia común variable. Primera experiencia en España. Anales de Pediatría (Barcelona) 70: 111-119.

12. Ochs HD, Gupta S, Kiessling, Nicolay U, Berger M (2006) Safety and efficacy of self- administered subcutaneous immunoglobulin in patients with primary immunodeficiency diseases. J Clin Immunol 26: 265-273.

13. Chapel HM, Spickett GP, Ericson D, Engl W, Eibl MM, et al. (2000) The comparison of the efficacy and safety of intravenous versus subcutaneous immunoglobulin replacement therapy. J Clin Immunol 20: 94-100.

14. Borte M, Bernatowska E, Ochs HD (2011) Efficacy and safety of home-based subcutaneous immunoglobulin replacement therapy in pediatric patients with primary immunodeficiencies. Clinical and Experimental Immunology 1-8.

15. Gardulf A, Hansen S, Johansson K (2005) Rapid subcutaneous IgG replacement therapy in children and adults-20 years of clinical experience. VII Meeting of the Spanish group for Primary Immunodeficiencies. Inmunología 24: 392-395.

16. Gardulf A, Nicolay U, Asensio O (2006) Rapid Subcutaneous IgG Replacement Therapy is Effective and Safe in Children and Adults with Primary Immunodeficiencies-A Prospective, Multi-National Study. J Clin Immunol 26 177-185. 
Citation: Liliana B, Andrea GR, Lorena R, Damacia DB, Gisela S, et al. (2014) Subcutaneous IgG Replacement Therapy by Push in 32 Patients with Primary Immunodeficiency Diseases in Argentine. Clin Exp Pharmacol 4: 148. doi:10.4172/2161-1459.1000148

17. Bezrodnik L, Gómez Raccio A, Belardinelli G (2013) Comparative Study of Subcutaneous vs. Intravenous IgG Replacement Therapy in Pediatric Patients with Primary Immunodeficiency Diseases: A Multi-center study in Argentina. J Clin Immunol 33

18. Shapiro R (2010) Subcutaneous immunoglobulin therapy by rapid push is preferred to infusion by pump: a retrospective analysis. J Clin Immunol 30: 301-307.

19. Conley ME, Notarangelo LD, Etzioni A (1999) Diagnostic criteria for primary immunodeficiencies. Representing PAGID (Pan-American Group for Immunodeficiency) and ESID (European Society for Immunodeficiencies). Clin Immunol 93: 190-197.

20. Sorensen RU, Leiva LE, Javier FC 3rd, Sacerdote DM, Bradford N, et al. (1998) Influence of age on the response to Streptococcus pneumoniae vaccine in patients with recurrent infections and normal immunoglobulin concentrations. J Allergy Clin Immunol 102: 215-221.

21. FDA (2008) Safety, efficacy, and pharmacokinetic studies to support marketing of immune globulin intravenous (human) as replacement therapy for primary humoral deficiency. Guidance for industry.

22. Gaspar J, Gerritsen B, Jones A (1998) Immunoglobulin replacement treatment by rapid subcutaneous infusion. Arch Dis Child 79: 48-51.
23. Björkander J, Chapel H, Spickett G (1998) Comparison of the efficacy and safety of immunoglobulin given subcutaneously versus intravenous immunoglobulin in the prevention of infection in patients with primary antibody deficiency syndromes. Mol Immunol 35: 11-12.

24. Berger M (2004) Subcutaneous immunoglobulin replacement in primary immunodeficiencies. Clin Immunol 112: 1-7.

25. Waniewski J, Gardulf A, Hammarström L (1994) Bioavailability of gammaglobulin after subcutaneous infusions in patients with common variable immunodeficiency. J Clin Immunol 14: 90-97.

26. Gustafson R, Hammarström L (2002) Subcutaneous immunoglobulin replacement therapy. Ellipse 318: 45-48.

27. Gardulf A, Nicolay U, Math D, Asensio O, Bernatowska E, et al. (2004) Children and adults with primary antibody deficiencies gain quality of life by subcutaneous IgG self-infusions at home. J Allergy Clin Immunol 114: 936-942.

28. Nicolay U, Kiessling P, Berger M, Gupta S, Yel L, et al. (2006) Healthrelated quality of life and treatment satisfaction in north american patients with primary immunodeficiency diseases receiving subcutaneous IgG self-infusions at home. J Clin Immunol 26: 65-72. 